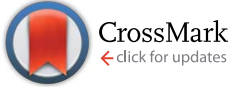

Cite this: RSC Adv., 2017, 7, 1634

\title{
Shear effects on the diffusive movement of oil in triacylglycerol networks
}

\author{
Nuria C. Acevedo, ${ }^{\text {a }}$ Bryce MacMillan, ${ }^{\mathrm{b}}$ Benedict Newling ${ }^{\mathrm{b}}$ \\ and Alejandro G. Marangoni ${ }^{\mathrm{C}}$
}

Oil migration is the foremost contributor to the quality loss of many high fat food products; thus, developing an understanding on how this phenomenon takes place in food systems is crucial for the food industry. Diffusion of triacylglycerols (TAG) through the fat network has often been modeled using simplified solutions to Fick's second law. However, a drawback of the use of diffusion models is the lack of sensitivity toward some microstructural characteristics of the matrix. This work reports the study of molecular and macroscopic oil diffusion coefficients of fat crystal networks using nuclear magnetic resonance measurements and from gravimetric determinations of oil loss, respectively. Blends of fully hydrogenated soybean oil (FHSO) in soybean oil (SO) were crystallized statically, under laminar shear rates of 30 and $240 \mathrm{~s}^{-1}$ at different wall temperatures $\left(-10,0,20{ }^{\circ} \mathrm{C}\right)$. Another batch of similar blends prepared with and without emulsifier was crystallized in a scraped surface heat exchanger. The results showed a good correlation between diffusion coefficients obtained using both methodologies only when samples exhibited low oil loss, particularly in blends crystallized statically or under mild-shearing conditions. This work suggests that although the Ziegleder model has great advantages and provides valuable insight into the oil migration in fat matrices, there remains a large need for further evaluating its suitability, principally in cases where crystalline structure is severely affected by processing conditions and where oil migration mechanisms other than diffusion may be involved.

Received 6th October 2016

Accepted 1st November 2016

DOI: $10.1039 / c 6 r a 24829 b$

www.rsc.org/advances

\section{Introduction}

In confectionary and many other high fat products, oil migration is a key contributor to product quality loss. Controlling the migration of liquid oil in a fat rich product is critical for maintaining an acceptable quality. Models that predict fat migration can be very useful to optimize manufacturing processes and guarantee the best quality of the final product for consumers. Diffusive transport is usually described using Fick's law, which relates the diffusive solute flux to the difference in liquid phase solute concentration between adjacent regions by means of a diffusion coefficient. In the last few years, several attempts have been made to model fat migration using simplified solutions to this Fick's second law., ${ }^{\mathbf{1 , 2}}$ However, a major concern regarding the use of diffusion models has been the lack of sensitivity toward other microstructural aspects of the matrix. ${ }^{3}$ Many findings have suggested that a capillary flow

${ }^{a}$ Department of Food Science and Human Nutrition, Iowa State University, 2312 Food Sciences Building, Ames, IA 50011-1054, USA. E-mail: nacevedo@iastate.edu; Tel: +1-515-294-3011; +1-515-294-5962

${ }^{b}$ MRI Centre, Department of Physics, University of New Brunswick, Fredericton, NB, CanadaE3B 5A3. E-mail: bnewling@unb.ca; bryce@unb.ca

${ }^{c}$ Guelph-Waterloo Physics Institute, Centre for Food \& Soft Materials Science, Department of Food Science, University of Guelph, 50 Stone Road East, Guelph, Ontario, Canada, N1G 2W1. E-mail: amarango@uoguelph.ca mechanism may play a role on the migration of oil in high fat products. ${ }^{3}$ For instance, Ziegler et $a l .{ }^{4}$ reported that oil migration is not well described by Fickean diffusion at all temperatures. Marty et al. ${ }^{5}$ suggested that both diffusion and capillary rise mechanisms might be active during oil migration through cocoa butter. However, it has recently been proposed that diffusion is the prevalent mechanism of oil migration: Ziegler ${ }^{6}$ reported that capillary action is too fast; within the order of seconds and hours to be considered in the timescale of oil migration wish is in the range of days or months. Furthermore, Altimiras et al. ${ }^{7}$ after solving Lucas-Washburn equation that describes the kinetics of capillary rise, obtained capillary curves which predicted oil migration with times 800 shorter than those observed experimentally, therefore indicating little role of capillary forces in oil migration. Thus, current simplified models are not adequate to reproduce experimental data because simplifying assumptions are not satisfied. Today, there is still the need to understand the exact mechanism of fat and oil migration through a crystallized network of lipids in order to aid the modelling of the structuring process and therefore allow the design of fat products with predictable and desirable oil binding capacity.

This paper addresses changes in molecular and macroscopic diffusion of oil in fat crystal networks crystallized under different conditions and attempts to evaluate the transport 
mechanism that determine these changes as well as their relationship with the physical and structural properties of the systems. Diffusivities were measured at both, the molecular and macroscopic level using two different approaches. Coefficients of diffusion measured by nuclear magnetic resonance $\left(D_{\text {mol }}\right)$ were compared to those calculated using the Fickean-based diffusion model $\left(D_{\text {eff }}\right)$ fitted to macroscopic oil loss data.

\section{Experimental}

\section{Materials}

Fully hydrogenated soybean oil (FHSO) and soybean oil (SO) were generously provided by Bunge Canada (Toronto, Canada). All chemicals and organic solvents were purchased from Fisher Scientific and Sigma-Aldrich (ON, Canada).

\section{Blend preparation}

Blends of FHSO and SO were prepared as reported by Acevedo et $a l .{ }^{8}$ Briefly, binary FHSO:SO blends were mixed in $45: 55$ $(\mathrm{w} / \mathrm{w})$ proportions. The mixtures were melted and held at $80{ }^{\circ} \mathrm{C}$ for $30 \mathrm{~min}$ to erase crystal memory. Then, the samples were crystallized for 30 minutes in a shear cell with a Searle configuration at 3 different wall temperatures $\left(-10{ }^{\circ} \mathrm{C}, 0{ }^{\circ} \mathrm{C}\right.$ and 20 $\left.{ }^{\circ} \mathrm{C}\right)$, and laminar shear rates $\left(0,30\right.$ and $\left.240 \mathrm{~s}^{-1}\right)$. After crystallization, the mixtures were kept at $20{ }^{\circ} \mathrm{C}$ for 48 hours to allow the material setting and subsequently stored at $4{ }^{\circ} \mathrm{C}$ until the moment of analysis.

Another set of FHSO:SO blends were prepared according to Acevedo and Marangoni. ${ }^{9}$ FHSO and SO were mixed at different ratios in order to formulate $40: 60 ; 30: 70$; and $20: 80 \mathrm{w} / \mathrm{w}$ FHSO:SO blends. Additionally, different emulsifiers in a concentration of $1 \% \mathrm{w} / \mathrm{w}$ were incorporated to $30: 70$ FHSO:SO blends. The emulsifiers added to the mixtures were Glyceryl Monostearate (GMS), Polyethylene Glycol Sorbitan Monostearate -Tween 60- (PGMS), Glyceryl Monopalmitate (GMP); Sorbitan Monopalmitate (SMP), Sodium Stearoyl Lactylate (SSL), phosphatidylcholine (P-CHOLINE), 75 BFP - mono and di glycerides from hydrogenated palm fat (BFP-75). Fat blends were crystallized in a pilot plant scale scraped surface heat exchanger or votator. After melted in the vessel and kept at $80{ }^{\circ} \mathrm{C}$ for $30 \mathrm{~min}$, fat blends were cooled down to $70{ }^{\circ} \mathrm{C}$. The molten mixture was pumped at a flow rate of $0.65 \mathrm{~kg} \mathrm{~min}^{-1}$ (39 $\mathrm{kg} \mathrm{h}^{-1}$ ) through the votator line. The tubing system between each unit and the outlets were well-insulated to avoid heat loss. In all experiments, the wall temperature of each unit was set to reach $\sim-2{ }^{\circ} \mathrm{C}$ in unit $\mathrm{A}, \sim 15{ }^{\circ} \mathrm{C}$ in unit $\mathrm{B}$ and $\sim 10{ }^{\circ} \mathrm{C}$ in unit $\mathrm{C}$. Fat blends were collected after passing through 2 scrapedsurface chiller (A and $\mathrm{C}$ units) and the agitated working unit (unit B) in a configuration ABC. After crystallization the samples were held at $20^{\circ} \mathrm{C}$ for 2 days to allow crystallization completion and subsequently stored at a refrigerator temperature $\left(4^{\circ} \mathrm{C}\right)$ until the moment of characterization.

\section{Solid fat content determination}

Crystallized samples were introduced into NMR glass tubes and stored for 24 hours at $4{ }^{\circ} \mathrm{C}$. Then, the tubes were incubated at the desired temperature for $30 \mathrm{~min}$ to allow a homogeneous distribution of temperature at the moment of measurement. Solid fat content (SFC) was measured by pulse nuclear magnetic resonance (p-NMR) using a Bruker Minispec spectrometer, (Bruker Optics Ltd., Milton, ON, Canada). The reported data corresponds to the average of at least five individual measurements.

\section{Polarized light microscopy}

Polarized light microscopy (PLM) was used to observe fat microstructure as described previously. ${ }^{9}$ Briefly, a definite amount of the crystallized sample plus soybean oil was weighed on a slide in order to maintain a 1:1 proportion; then the mixture was homogeneously spread in all directions and cover glass was carefully laid over the fat to remove air and complete spreading the fat. Samples were imaged using a Leica DM RXA2 microscope with polarized light (Leica Microsystems, Richmond Hill, Canada) and equipped with a CCD camera (Q Imaging Retiga 1300, Burnaby, BC, Canada). All images were acquired using a $40 \times$ objective lens (Leica, Germany). The camera was set for autoexposure. Openlab 6.5.0 software (Improvision, Waltham, MA, USA) was used to acquire images. Focused images were stored as uncompressed 8 bit (256 grays) grayscale TIFF files with a $1280 \times 1024$ spatial resolution. Five images were captured from each of the five replicates prepared.

Microstructural analysis was carried out by image analysis employing the Adobe Photoshop CS 3 software (Adobe Systems Inc., San Jose, California, USA) and filters from the Fovea Pro 4.0 software (Reindeer Graphics, Inc., Asheville, NC, USA). A manual threshold was applied to all the pictures to convert the grayscale images to binary images, in order to discriminate between features and background and to measure the features sizes. The microstructural elements were determined using the filter tools included in the Fovea Pro software.

\section{Cryogenic transmission electron microscopy (Cryo-TEM)}

In order to discard oil fraction and favor single crystals observation, fat blends were treated as reported previously by Acevedo and Marangoni. ${ }^{\mathbf{1 0 , 1 1}}$ Five microliters of the obtained dispersion were placed on a copper grid with perforated carbon film (Canemco-Marivac, Quebec, Canada), and excess liquid was blotted automatically for $2 \mathrm{~s}$ using filter paper. A staining aqueous solution of $2 \%$ of uranyl acetate was used to enhance contrast. Subsequently, the sample was transferred to a Cryo holder (Gatan Inc., Pleasanton, CA, USA) for direct observation at $-176{ }^{\circ} \mathrm{C}$ in a FEI Tecnai G2 F20 Cryo-TEM operated at $200 \mathrm{kV}$ in low dose mode (Eidhoven, The Netherlands). Images were taken using a Gatan 4k CCD camera. Micrographs were stored and analyzed using DigitalMicrograph ${ }^{\mathrm{TM}}$ software (USA). Image J 1.42q software (USA) was employed for a semiautomatic analysis procedure.

\section{Oil loss determination}

Oil migration studies were performed according with the technique described previously by Dibildox-Alvarado et al. ${ }^{12}$ Once crystallized, fat blends were molded in discs of $22 \mathrm{~mm}$ diameter 
and $3.2 \mathrm{~mm}$ of thickness using polyvinyl chloride (PVC) molds and then transferred to filter papers (Whatman \# 5, $110 \mathrm{~mm}$ diameter). The amount of oil that each sample (prepared as discs) lost to filter papers was determined by the difference in weight of the filter papers before and after placing the fat disc on the paper for 24 hours at $20^{\circ} \mathrm{C}$. A "blank" filter paper was included in all experiments to account for the effects of the treatments on the paper itself such as the influence of the humidity of storage environment. Filter papers must be large enough in order to avoid the paper saturation with oil during the period of measurement. An average and standard deviation of at least five replicates (five separate disks on individual filter papers) is reported. Oil migration (\%) was calculated as:

$$
\mathrm{OL}(\%)=\frac{\text { wt paper }(24 \mathrm{~h})-\mathrm{wt} \text { paper }(0 \mathrm{~h})}{\mathrm{wt} \text { paper }(0 \mathrm{~h})} \times 100
$$

\section{Nuclear magnetic resonance}

Nuclear magnetic resonance (NMR) experiments were performed on a Tecmag (Houston, TX, USA) Apollo console equipped with a Nalorac 2.4-T 32-cm i.d. horizontal bore superconducting magnet (Nalorac Cryogenics, Martinez, CA, USA). Sample excitation and signal detection were both accomplished with a homebuilt radiofrequency (RF) sensor (an eight-rung birdcage coil ${ }^{13}$ ), $4 \mathrm{~cm}$ in diameter and $10 \mathrm{~cm}$ in length, driven by a $2 \mathrm{~kW} 3445 \mathrm{RF}$ amplifier (American Microwave Technology, Brea, CA, USA). Magnetic field gradients for diffusion sensitization were generated by supplying current to a 200 mm-i.d. homebuilt coil winding ${ }^{14}$ from a Techron (Elkhart, IN, USA) 8710 amplifier, providing for maximum gradient strengths of $900 \mathrm{mT} \mathrm{m}^{-1}$.

The series of RF and magnetic field gradient applications (the NMR pulse sequence) used to measure a diffusion coefficient is illustrated in Fig. 1. In the diagram, each line indicates the history of magnetic field gradient application (G) or RF application (r.f.) and detection (rx). The fat sample becomes magnetized when it is placed in the superconducting magnet, with the net sample magnetization aligned along the main magnetic field (conventionally designated the $z$-axis). Pulses of RF labeled $90^{\circ}$ or $180^{\circ}$ have sufficient amplitude and duration to rotate the sample magnetization, about the applied RF field,

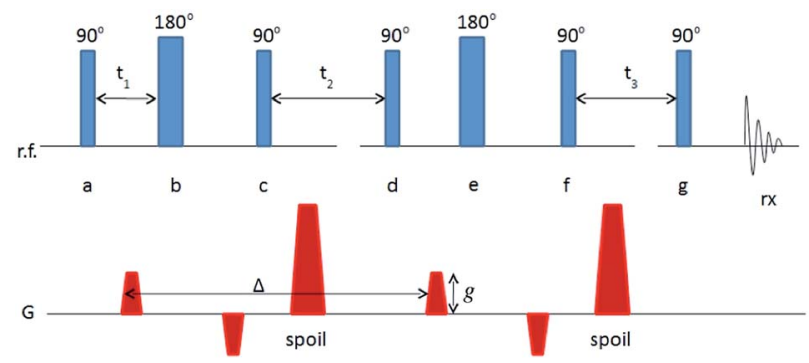

Fig. 1 Pulse sequence diagram for the Cotts-13-interval diffusion sensitised scheme. The top line is a history of radiofrequency application $(\mathrm{a}-\mathrm{g}$ ) and detection ( $\mathrm{rx}$ ). The bottom line is a history of magnetic field gradient application. through the angle shown. The two pairs of magnetic field gradients of amplitude $g$ sensitize the net sample magnetization (and, hence, the amplitude of detected signal) to diffusion during the interval $\Delta$. Broadly, the greater the diffusion coefficient, the greater the attenuation of detected signal for a given $g$. This particular use of up-down pairs of magnetic field gradient, combined with $180^{\circ} \mathrm{RF}$ pulses (known as the Cotts 13-interval sequence) eliminates any incidental signal attenuation caused by heterogeneities in the magnetic properties of the sample. ${ }^{15}$ The magnetic field gradient pulses labeled "spoil" destroy unwanted sample magnetization, which can be generated by the sequence of RF pulses. Unwanted magnetization is further suppressed by variation of the relative phases of the RF pulses (phase cycling) according to the cogwheel scheme. ${ }^{\mathbf{1 6}}$

For each fat sample, the amplitude of $g$ was varied in 20 steps from 0 to a maximum of 600 or $900 \mathrm{mT} \mathrm{m}^{-1}$. The decay of the ratio, $S_{\mathrm{N}}=S / S_{0}$, of detected signal to that at $g=0$ was fit, using SigmaPlot (Systat Software Inc., San Jose, CA, USA) with:

$$
S_{\mathrm{N}}=\exp \left(-\gamma^{2} \delta^{2} g^{2}\left[4 t_{2}+6 t_{1}-2 \delta / 3\right] D\right.
$$

when the decay was clearly monoexponential. Here $\gamma$ is the gyromagnetic ratio of ${ }^{1} \mathrm{H}\left(2.675 \times 108 \mathrm{rad}\left(\mathrm{s}^{-1} \mathrm{~T}^{-1}\right)\right), \delta$ is the area of one half of the diffusion-sensitizing up-down gradient pair and other timing parameters. The fit parameter, $D$, is the free molecular self-diffusion coefficient of the ${ }^{1} \mathrm{H}$ in the sample. ${ }^{17}$ A sample fit is shown in Fig. 2.

A variety of more complex models for the signal were assessed for non-exponential decays (see results), but the consistently best and most stable fit was to the function:

$$
\begin{aligned}
S_{\mathrm{N}}= & \exp \left(-4 \gamma^{2} \delta^{2} g^{2}\left[t_{2}+2 t_{1}-2 \delta / 3\right] D_{2}\right) \\
& \times \int_{0}^{1} \exp \left(4 \gamma^{2} \delta^{2} g^{2}\left[t_{2}+2 t_{1}-2 \delta / 3\right] D_{2} x^{2}\right)
\end{aligned}
$$

which was expanded as a series in 8 terms and again fit in SigmaPlot. This function models the signal decay caused by

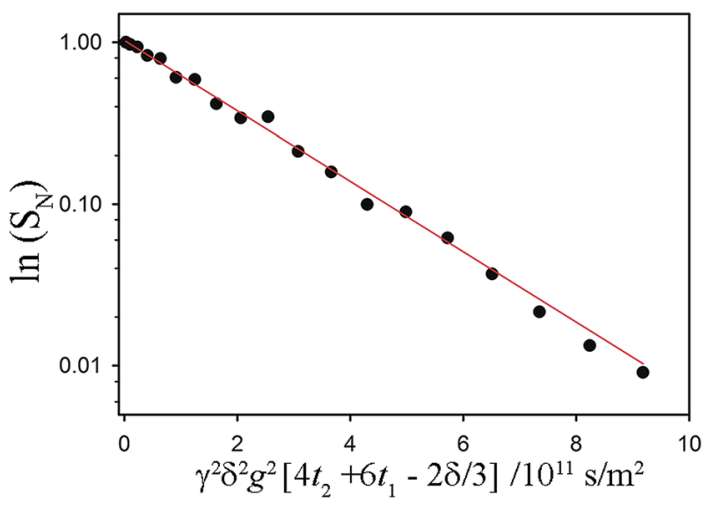

Fig. 2 Sample fit to a non exponential NMR signal decay associated with free diffusion of the mobile ${ }^{1} \mathrm{H}$ in the fat sample. The acquisition time for these data was 13 minutes (with 16 coadded scans to improve signal-to-noise ratio). Referring to the timings indicated in the pulse sequence diagram, $\delta=7.6 \mathrm{~ms}, t_{2}+2 t_{1}=\Delta=61 \mathrm{~ms}$. The maximum value of $g$ was $900 \mathrm{mT} \mathrm{m}^{-1}$. The fat sample was $30: 70$ ABC 1\% SSL (votator crystallization) and the data were taken at $4{ }^{\circ} \mathrm{C}$. 


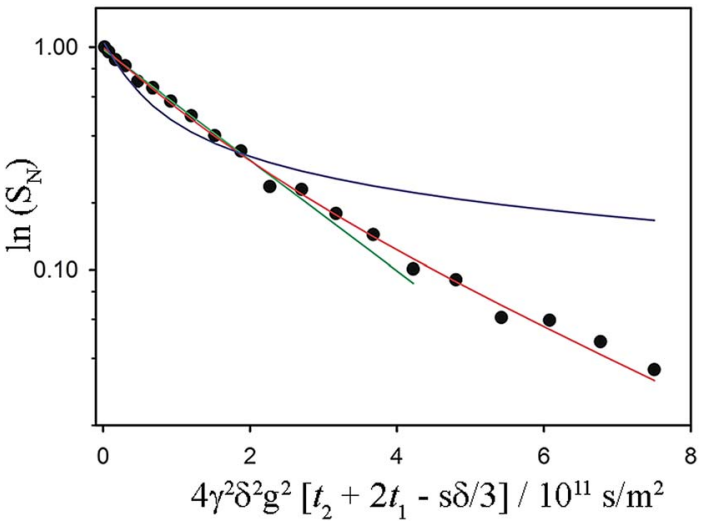

Fig. 3 Sample fits to a non-exponential NMR signal decay associated with restricted diffusion of the mobile ${ }^{1} \mathrm{H}$ in a fat sample. The acquisition time for these data was 13 minutes (with 16 coadded scans to improve signal-to-noise ratio). Referring to the timings indicated in the pulse sequence diagram, $\delta=7.6 \mathrm{~ms}, t_{2}+2 t_{1}=\Delta=60 \mathrm{~ms}$. The maximum value of $g$ was $900 \mathrm{mT} \mathrm{m}^{-1}$. The fat sample was $45: 55$ (laminar shear crystallizer), shear rate $=200 \mathrm{~s}^{-1}$, wall temperature $=$ $20^{\circ} \mathrm{C}$ and the data were taken at $18^{\circ} \mathrm{C}$. The green and the blue lines are attempted fits using a free diffusion model (like that used in Fig. 2) and a one-dimensional diffusion model (diffusion confined to randomly oriented tubes) respectively. Clearly preferable is the red fit line, which models diffusion restricted to two dimensions in a sample consisting of many randomly oriented lamellar domains.

diffusion restricted to two dimensions. Such diffusion would be typical of a sample composed of many lamellar domains, randomly oriented with respect to the magnetic field gradient. ${ }^{17}$ The fit parameter is the coefficient of diffusion, $D_{2}$, parallel to the plane of the confining lamellae within each domain (this being much greater than the diffusion coefficient perpendicular to their plane). An example of non-exponential fit is shown in Fig. 3.

\section{Statistical analysis}

Data were processed using GraphPad Prism 5 software (GraphPad Software, Inc., San Diego, CA, USA). Reported values correspond to means and standard errors of the determinations. Statistical analysis was performed by one-way ANOVA ( $p<$ 0.001 ) using Tukey's multiple comparisons as post-test $(p<$ $0.005)$.

\section{Results and discussion}

The macroscopic and functional properties of fats, such as mechanical strength, texture, mouthfeel, and other sensory characteristics strongly depend on the structural organization at the nano- and meso-scale. ${ }^{18,19}$ It was recently discovered that crystalline nanoplatelets (CNP) with a characteristic length scale of approximately $100 \mathrm{~nm}$ represent the fundamental structural unit of fat crystal networks. ${ }^{10}$ Thus, a bulk fat as we know it, is comprised by a spherulitic colloidal network of polycrystals (Fig. 4A and B) at the microstructural level, which is in turn the result of CNP assembled from crystalline lamellae of triglycerides (Fig. 4C). ${ }^{20}$ Furthermore, fat crystalline networks

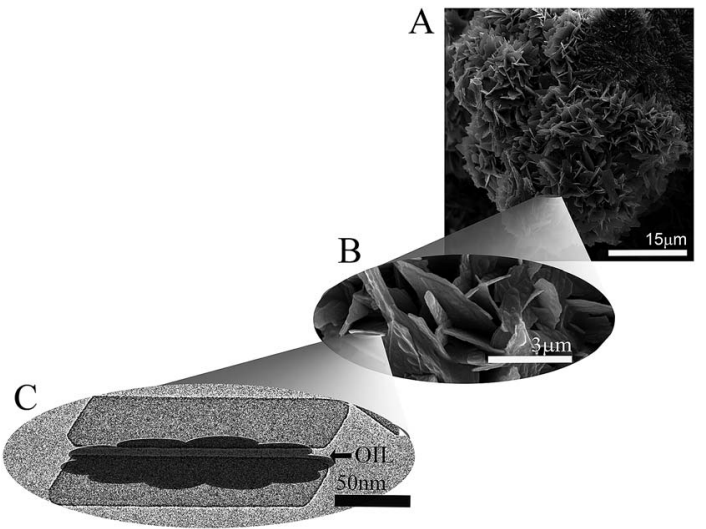

Fig. 4 Fat structure at different length scales. SEM images showing a spherulitic colloidal network of polycrystals and mesocrystals (A and B). TEM image depicting primary crystalline nanoplatelets composed of triglycerides lamellae (C)

contain liquid oil trapped within. The distribution of liquid oil within a fat crystal network and the ability of the matrix to entrap and bind liquid oil are essential for its stability, as well as for its mechanical and thermal properties. Liquid oil is distributed in the space between crystals and crystal aggregates, thus the surface of the CNP and aggregates is in complete contact with the oil (Fig. 4C).

The mechanisms controlling oil migration throughout fat crystal networks are still uncertain, thus its study is a topic of continuous debate. Furthermore, it is worth discussing the fact that the amount of oil lost from the fat matrix is the result of both, local and global oil migration, in other words, the result of molecular and macroscopic oil diffusion. This raises doubts not only on the contribution of strongly and loosely bound and free oil to the overall mechanism of oil migration but also on the relationship, if any, between both types of oil movement and the system properties. Ziegleder et al. ${ }^{\mathbf{1 , 2 1}}$ proposed an equation to model oil migration in fats, based on Fickean diffusion and that can be simplified for a slab as:

$$
\frac{m_{t}}{m_{\max }}=\frac{\sqrt{D_{\text {eff }} t}}{l}
$$

with $m_{\max }$ and $m_{t}$ being the maximum migration and the mass migrated at time $t$, respectively. $l$ is the length corresponding to the maximum distance traveled by the oil front; in this case the width of the fat disc $(3.2 \mathrm{~mm})$ and $D_{\text {eff }}$ is the effective diffusion coefficient obtained from the macroscopic oil loss. Additionally, since the model is asymptotic for long times; $m_{\max }$ can be obtained as:

$$
m_{\max }=\left(1-\frac{\mathrm{SFC}}{100}\right) \times m_{\mathrm{disc}}
$$

where $m_{\text {disc }}$ is the initial weight of the fat disc used for the oil migration experiments. Many parameters can affect oil diffusivity through the network, such as processing conditions and chemical composition of the system. The calculated diffusion coefficients $\left(D_{\text {eff }}\right)$ as well as the obtained OL values in $45: 55$ FHSO:SO blends subjected to a laminar shear crystallization at 
0,30 and $240 \mathrm{~s}^{-1}$ using wall temperatures of $-10,0$ and $20{ }^{\circ} \mathrm{C}$ are represented in Fig. 5A and B, respectively. As expected, both $D_{\text {eff }}$ values and OL increased significantly as the laminar shear rate used during crystallization was greater, signifying that shear affects the structure of the system in such a way that oil loss (diffusion of liquid oil from the crystal network) is enhanced. Furthermore, at each determined shear rate, the increase in wall temperature led to higher values of $D_{\text {eff }}$ and OL, however the temperature influence was found to be not as important as the laminar shear rate. For instance, at $30 \mathrm{~s}^{-1}$ there was a two fold increase in $D_{\text {eff }}$ when changing the wall temperature from 0 to $20{ }^{\circ} \mathrm{C}$; whereas at $0{ }^{\circ} \mathrm{C} D_{\text {eff }}$ was at least 3 orders of magnitude higher when changing the shear rate from $0 \mathrm{~s}^{-1}$ to $30 \mathrm{~s}^{-1}$ and $240 \mathrm{~s}^{-1}$. It is also interesting to note that the $D_{\text {eff }}$ values are in line with values existing in the literature for similar fat systems., ${ }^{\mathbf{1 , 5 2 - 2 5}}$ Nevertheless, the results obtained in this work are opposite to those reported by Maleky et $a l .{ }^{25}$ when samples were subjected to shearing process; these authors described a reduction of oil migration in a cocoa butter matrix crystallized in a laminar shear fashion. Nonetheless, they worked with a significantly more complex system in composition and with a significantly higher shear rate $\left(340 \mathrm{~s}^{-1}\right)$ which translates into a different crystalline network organization and therefore a different oil movement within the sample.
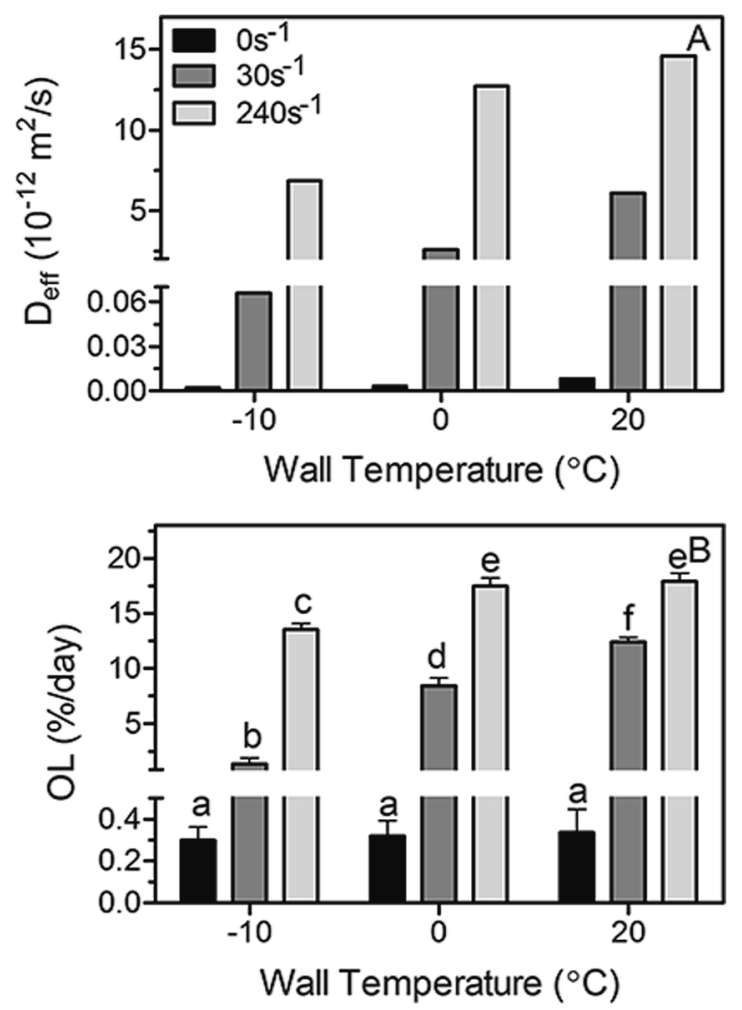

Fig. 5 Diffusion coefficients ((A), $\left.D_{\text {eff }}\right)$ calculated using the simplified model of Ziegleder et al. ${ }^{1}$ and oil loss (OL) values (B) obtained for nonsheared and sheared blends of 45 : 55 Fully Hydrogenated Soybean Oil (FHCO) and Soybean Oil (SO). Bars and error bars represent mean and standard deviation values. Different letters represent statistically significant different between values $(p<0.05)$.
Diffusion coefficients $\left(D_{\text {mol }}\right)$ measured at $4{ }^{\circ} \mathrm{C}$ and $18{ }^{\circ} \mathrm{C}$ by NMR for the same fat blends, as well as the relationship between them are plotted in Fig. 6A-C, respectively. Not surprisingly, $D_{\text {mol }}$ measured at $18{ }^{\circ} \mathrm{C}$ are higher than those obtained at $4{ }^{\circ} \mathrm{C}$ owed to the increase in molecular mobility and the amount of energy available for diffusion. However, although minor differences between $D_{\text {mol }}$ magnitudes exist at both temperatures, similar trends with high correlations (Fig. 6C) can be observed at the studied shear rates-wall temperatures combinations. The results clearly demonstrate that similar assumptions can be made at both measured temperatures, thus
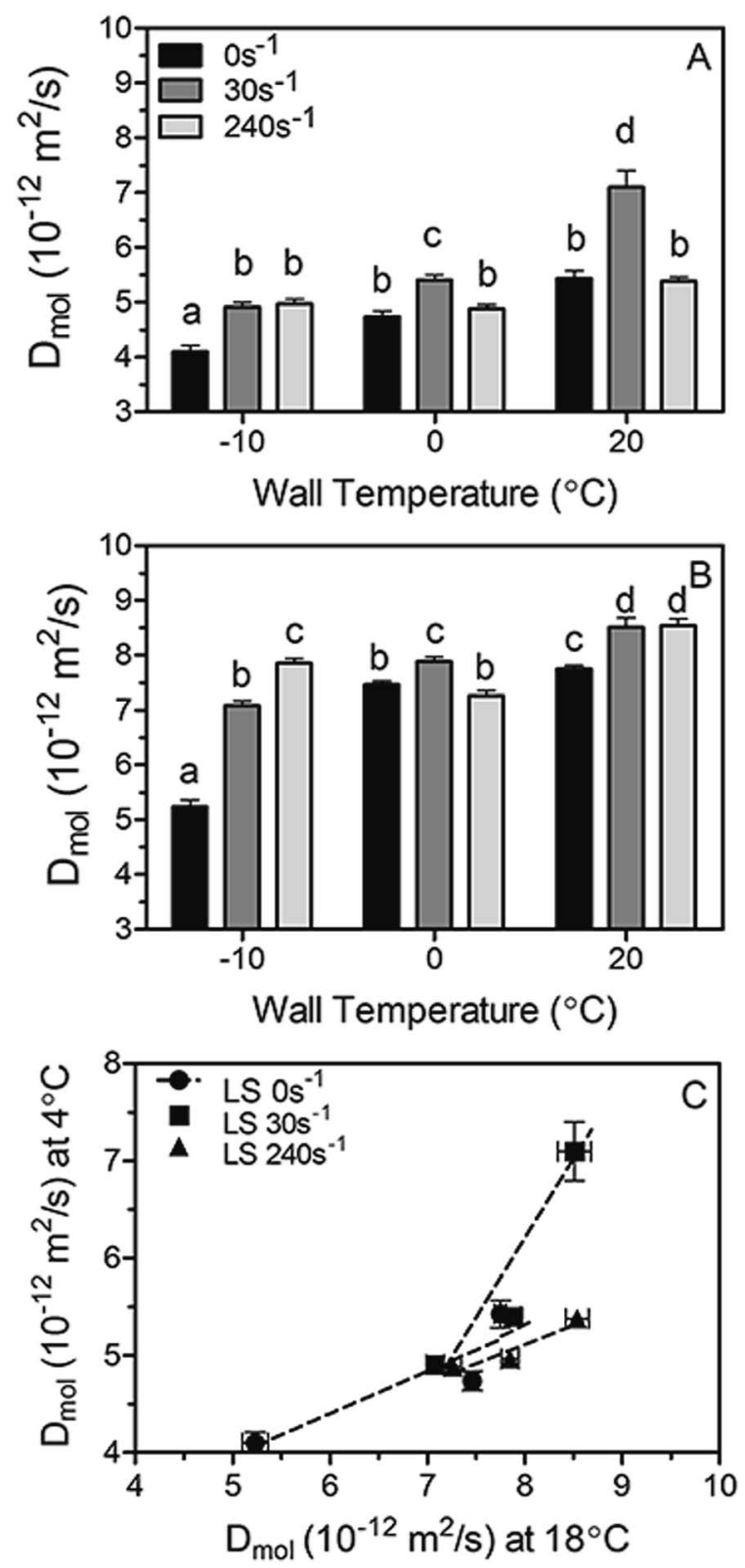

Fig. 6 Diffusion coefficients $\left(D_{\text {mol }}\right)$ measured by NMR at $4{ }^{\circ} \mathrm{C}(\mathrm{A})$ and $18^{\circ} \mathrm{C}(\mathrm{B})$ and correlation between them (C) obtained for non-sheared and sheared blends of 45 : 55 Fully Hydrogenated Soybean Oil (FHCO) and Soybean Oil (SO). Bars and error bars represent mean and standard deviation values. Different letters indicate statistically significant differences $(p<0.05)$. 
only diffusion coefficient determined by $\mathrm{NMR}$ at $18{ }^{\circ} \mathrm{C}$ will be considered and discussed in the following sections of this work.

Overall, $D_{\mathrm{mol}}$ is found to increase when increasing the shear rate and wall temperature during crystallization. Exceptions to this tendency can be observed upon shearing at $240 \mathrm{~s}^{-1}$, in particular at 0 and $20^{\circ} \mathrm{C}$ where the diffusivity $\left(D_{\text {mol }}\right)$ showed to be similar or inferior to the one observed at $30 \mathrm{~s}^{-1}$. Interestingly, the different trends observed between $D_{\text {eff }}$ (Fig. 5) and $D_{\text {mol }}$ (Fig. 6) at the highest shear rate studied can be explained by the fact that the diffusion coefficients obtained by both methods are a measure of essentially different phenomena occurring at different structural length scales. $D_{\text {eff }}$ is determined from the measurement of the macroscopic amount of oil lost by the fat sample; meanwhile $D_{\text {mol }}$ is related to the molecular mobility of oil within the fat matrix. It is most likely that a laminar shear rate of $240 \mathrm{~s}^{-1}$ with wall temperatures of 0 and $20{ }^{\circ} \mathrm{C}$, where shearing can affect more severely the crystallizing sample, induced a large change in the architecture of the matrix which resulted in the macroscopic loss of oil; nevertheless molecular and local oil mobility within the matrix is not affected in the same way. It is evident that perhaps in intensely sheared fat crystal networks other phenomena besides diffusion come into play which leads to high values of oil loss and therefore an overestimation of the diffusion coefficient $D_{\text {eff. }}$

An attempt was made to correlate $D_{\text {eff }}$ and $D_{\text {mol }}$, as displayed in Fig. 7. While both diffusivities were within the $10^{-15}$ to $10^{-11}$ $\mathrm{m}^{2} \mathrm{~s}^{-1}$ range and therefore comparable to previous reported values; ${ }^{\mathbf{1 , 5 2 2 - 2 5}}$ much higher $D_{\text {mol }}$ values (up to 3 orders of magnitude) were observed for samples processed under static conditions compared to $D_{\text {eff }}$. This is probably because there is a limit on the sensitivity of the method used to obtain $D_{\text {eff }}$ when OL values are very low, as occurs in the case of samples crystallized statically.

Furthermore, based on the obtained linear correlation coefficients values $\left(R^{2}\right) D_{\text {eff }}$ and $D_{\text {mol }}$ appear to be well correlated to each other under static and mild shearing conditions, with $R^{2}$ of 0.613 and 0.974 , respectively. As expected, an exception was found for crystallization under a laminar shear rate of $240 \mathrm{~s}^{-1}$ where no relationship can be detected between both diffusion coefficients. As discussed previously, these results probably denote a change on the crystal network structure induced by high shear forces that reflects on the macroscopic diffusivity which in turn encompasses not only mechanistic but also microstructural effects. Hence, previous results point out that in spite of the fact that the Ziegleder's expression is a simple formula to model the oil migration process, ${ }^{\mathbf{1}}$ it is fairly well correlated with the molecular approach to oil mobility determined by NMR. However, special considerations should be taken into account in circumstances where oil movement though the matrix may not be strictly diffusive, such as when structural features are severely altered by processing conditions.

To confirm the above conjecture, similar fat blends crystallized in a scraped surface heat exchanger, where turbulent high shear fields are applied, were analyzed on purpose for comparison. As expected, the plot $D_{\text {mol }} v s$. $D_{\text {eff }}$ for the samples exhibited a random dispersion, particularly at high values as
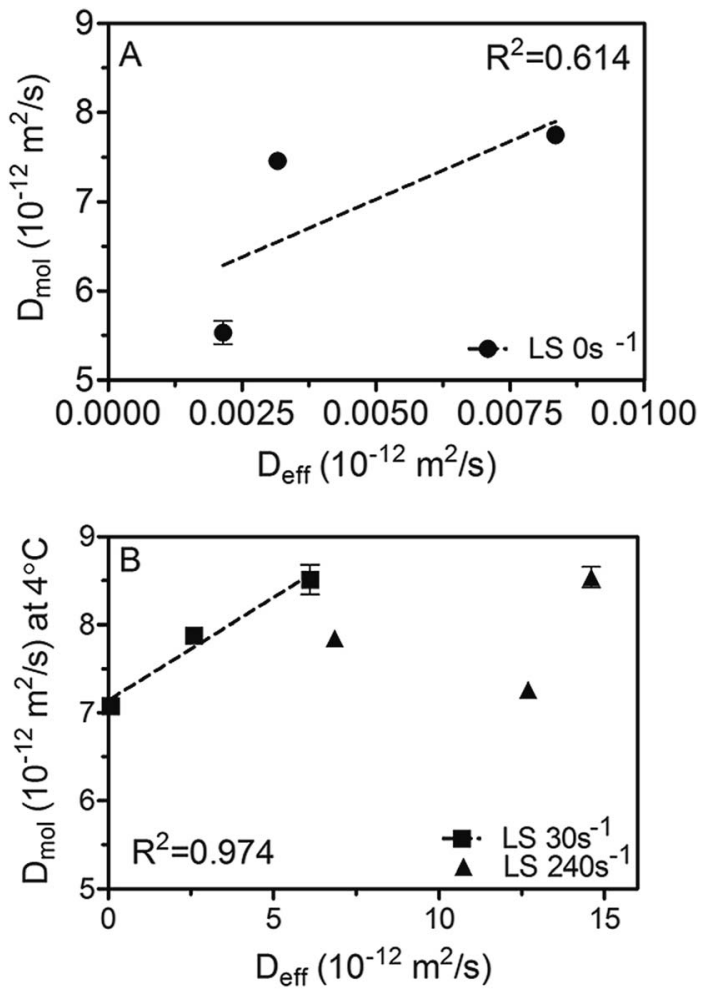

Fig. 7 Correlation between diffusion coefficients measured by NMR at $18^{\circ} \mathrm{C}\left(D_{\text {mol }}\right)$ and those calculated using the simplified model of Ziegleder et al. ${ }^{1}\left(D_{\text {eff }}\right) .45$ : 55 Fully Hydrogenated Soybean Oil (FHCO) and Soybean Oil (SO) non-sheared samples (A) and blends crystallized under laminar shear (B).

shown in Fig. 8A. As previously discussed and observed in Fig. 5, high $D_{\text {eff }}$ correspond to high OL values. The possible reasons for the discrepancy between $D$ values are the severe processing conditions the samples were exposed to during crystallization which led to a significant breakage of the structural features and thus, an oil movement in the matrix that is not merely diffusive. Both $D$ values exhibit a linear correlation only at low magnitudes. It should be noted that the diffusive regime is observed up to a specific value of OL. From approximately $3 \%$ OL and above the movement of oil is not only driven by diffusion (Fig. 8B). Instead, it is possible that oil transport within the fat matrix is mediated by convection currents. Thus, analysis of additional factors should be considered when a quantitative $D_{\text {eff }}$ determination is required in highly sheared samples.

In order to further examine the correlation between structural properties and oil transport, we plotted $D_{\text {mol }}$ as a function of SFC and crystal size (Fig. 9A and B). $D_{\text {mol }}$ obtained for all the samples show an inverse linear relationship (with relatively high correlation coefficients) with the SFC (\%) and the equivalent diameter of the mesocrystals present (Fig. 9A and B, respectively). Not surprisingly, the higher the amount of solid material in the network, the lower the oil movement, which in general translates into an increase in the ability of the fat matrix to bind liquid oil. Similarly, Ziegleder et al. ${ }^{1}$ reported a strong inverse dependence of $D$ with the amount of solid fat in chocolate. Acevedo et al.,$^{8}$ observed the same tendency when 

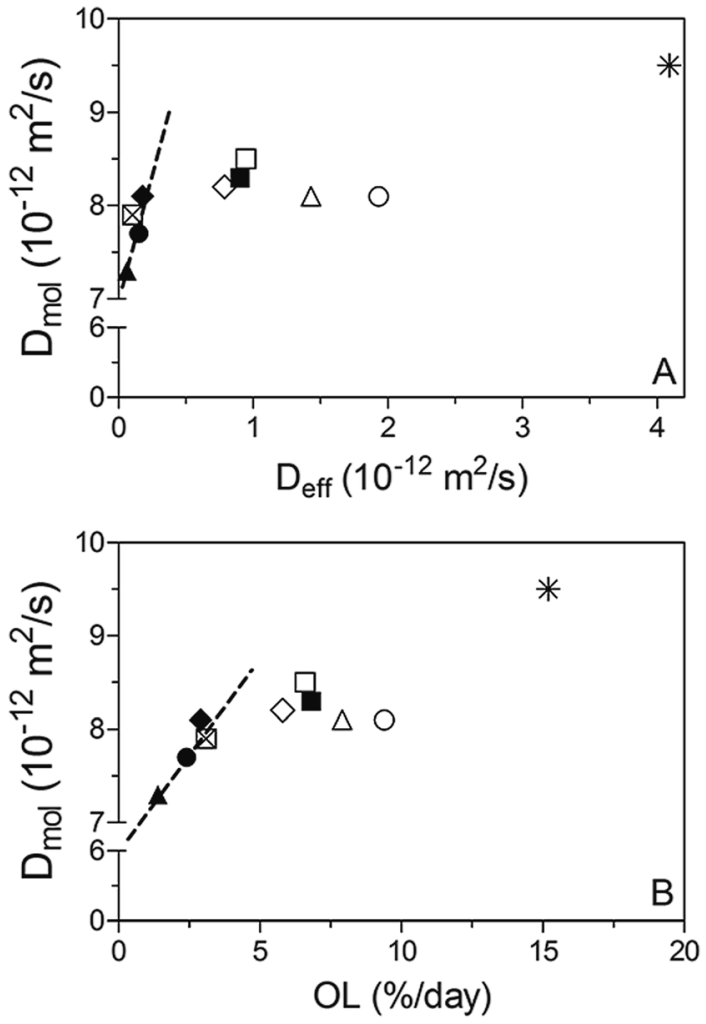

Fig. $8 D_{\text {mol }}$ obtained by NMR at $18^{\circ} \mathrm{C}$ as a function of $D_{\text {eff }}(\mathrm{A})$ and $\mathrm{OL}$ (B) for fat blends crystallized in a scraped surface heat exchanger or votator with an ABC configuration: $20: 80 \mathrm{FSHO}: \mathrm{SO}(\boldsymbol{\square}) ; 30: 70$ FSHO:SO (O); $30: 70$ FSHO:SO-1\% SMP $(*) ; 30: 70$ FSHO:SO-1\% SSL $(O) ; 30$ : 70 FSHO:SO-1\% PGMS ( $\square)$; 30 : 70 FSHO:SO-1\% BFP $(\diamond)$; 30 : 70 FSHO:SO-1\% GMS ( $)$; 30 : 70 FSHO:SO-1\% P-choline $(\triangle)$; $30: 70$ FSHO:SO-1\% GMP ( $\otimes) ; 40: 60$ FSHO:SO.
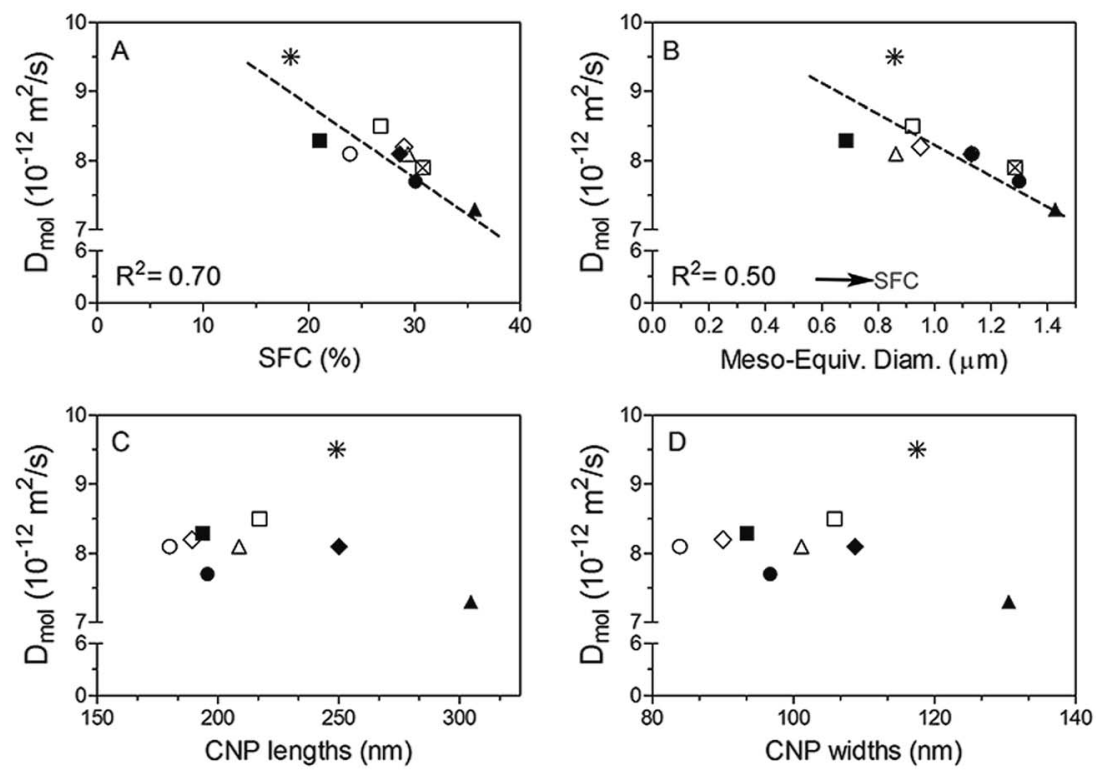

Fig. $9 D_{\text {mol }}$ obtained by NMR at $18{ }^{\circ} \mathrm{C}$ as a function of SFC (A), meso-equivalent diameter (B), crystal nanoplatelet lengths (C) and widths (D) for fat blends crystallized in a scraped surface heat exchanger or votator with an ABC configuration: $20: 80 \mathrm{FSHO}: \mathrm{SO}$ ( $\boldsymbol{\square}$ ); $30: 70 \mathrm{FSHO}$ :SO (O); 30 : 70 FSHO:SO-1\%SMP (*); 30 : 70 FSHO:SO-1\%SSL (O); $30: 70$ FSHO:SO-1\%PGMS ( $\square)$; $30: 70$ FSHO:SO-1\%BFP $(\diamond) ; 30: 70$ FSHO:SO-1\% GMS $(\diamond) ; 30: 70$ FSHO:SO-1\% P-choline $(\triangle)$; $30: 70$ FSHO:SO-1\% GMP $(\otimes)$; $40: 60$ FSHO:SO $(\Delta)$. 
$D_{\text {mol }}$ and $D_{\text {eff }}$ are not well correlated in many of the analysed samples as previously stated.

For further assessment of the relationship between diffusivity and system's structural properties, $D_{\text {mol }}$ values were plotted as a function of crystal nano-platelets (CNP) lengths (Fig. 9C) and widths (Fig. 9D) obtained by Cryo-TEM analysis. No significant correlation between CNP sizes and $D_{\text {mol }}$ was found. These results clearly show that fat crystal nanostructure, i.e. platelet sizes, do not seem to play a governing role in the mechanism of liquid TAG diffusion through a crystalline fat matrix; at least at the local or molecular level. Structural features, not only at the nanoscale but also at higher length scales are most likely strongly affected by processing conditions in the scraped surface heat exchanger. Additionally, the fact that an inverse correlation was observed between mesocrystal size and diffusivity leads us to hypothesize that other factors not considered yet are at play to explain oil migration in fat crystal networks.

Based on these findings, it is not surprising that a lack of correlation between $D$ values calculated using the Ziegleder approach $^{\mathbf{1}}$ and NMR were found, particularly in highly sheared materials. It is likely that strong alterations in the structure and permeability of the fat matrix occurred upon crystallization under laminar shear rates of $240 \mathrm{~s}^{-1}$ and in a scraped surface heat exchanger are responsible for the observed discrepancies. As mentioned earlier, perhaps the macroscopic oil leakage from the crystal network involves an additional contribution to that of molecular diffusion.

\section{Conclusions}

Two different approaches have been used to obtain diffusion coefficients and evaluate their relationship with each other and with physical and structural parameters of fat crystal networks. Interestingly, a significant correlation between $D_{\text {eff }}$, obtained through the Ziegleder model and $D_{\text {mol }}$ measured by NMR was observed only at low OL values; i.e. under static of mild shearing conditions during crystallization of the fat blends. On the contrary, no correlation between $D_{\text {mol }} v s$. $D_{\text {eff was found upon }}$ crystallization under high shear fields. This can be attributed in part to the contribution of other factors, particularly in cases where crystalline structure is drastically affected by processing conditions.

The results reported in this study are important since it has been verified that oil migration in highly sheared fat samples is not fully diffusive in nature. The assumption that the mechanism of oil migration is purely diffusive should be reconsidered. It is evident that further work needs to be carried out to fully describe and understand the behaviour of oil as it migrates through the a crystalline mass.

\section{Acknowledgements}

The authors acknowledge The Natural Sciences and Engineering Research Council of Canada and Advanced Foods and Materials network for the financial support.

\section{References}

1 G. Ziegleder, C. Moser and J. Geier-Greguska, Fett/Lipid, 1996a, 98, 196-199.

2 Y. J. Choi, K. L. McCarthy, M. J. McCarthy and M. H. Kim, Appl. Magn. Reson., 2007, 32, 205-220.

3 J. M. Aguilera, M. Michel and G. Mayor, J. Food Sci., 2004, 69, 167-174.

4 G. R. Ziegler, A. Shetty and R. C. Anantheswaran, Manuf. Confect., 2004, 84, 118-226.

5 S. Marty, K. Baker, E. D. Alvarado and A. G. Marangoni, Food Res. Int., 2005, 38, 1189-1197.

6 G. R. Ziegler, Science and Technology of Enrobed and Filled Chocolate, Confectionary and Bakery Products, CRC Press, Boca Raton, FL, 2009.

7 P. Altimiras, L. Pyle and P. Bouchon, J. Food Eng., 2007, 80, 600-610.

8 N. C. Acevedo, J. M. Block and A. G. Marangoni, Faraday Discuss., 2012, 158, 171-194.

9 N. C. Acevedo and A. G. Marangoni, Food Biophys., 2014, 9, 368-379.

10 N. C. Acevedo and A. G. Marangoni, Cryst. Growth Des., 2010, 10, 3327-3333.

11 N. C. Acevedo and A. G. Marangoni, Cryst. Growth Des., 2010, 10, 3334-3339.

12 E. Dibildox-Alvarado, J. Neves Rodrigues, L. A. Gioielli, J. F. Toro-Vazquez and A. G. Marangoni, Cryst. Growth Des., 2004, 4, 731-736.

13 C. E. Hayes, W. A. Edelstein, J. F. Schenck, O. M. Mueller and M. Eash, J. Magn. Reson., 1985, 63, 622-628.

14 K. Deka, M. B. MacMillan, A. V. Ouriadov, I. V. Mastikhin, J. J. Young, P. M. Glover, G. R. Ziegler and B. J. Balcom, J. Magn. Reson., 2006, 178, 25-32.

15 R. M. Cotts, M. J. R. Hoch, T. Sun and J. T. Marker, J. Magn. Reson., 1989, 83, 252-266.

16 M. H. Levitt, P. K. Madhu and C. E. Hughes, J. Magn. Reson., 2002, 155, 300-306.

17 P. T. Callaghan, Principles of Nuclear Magnetic Resonance Microscopy, Clarendon Press, Oxford, 1991.

18 D. Rousseau and A. G. Marangoni, Food Res. Int., 1999, 31, 381-388.

19 J. M. deMan and A. M. Beers, J. Texture Stud., 1987, 18, 303318.

20 N. C. Acevedo, F. Peyronel and A. G. Marangoni, Curr. Opin. Colloid Interface Sci., 2011, 16, 374-383.

21 G. Ziegleder, C. Moser and J. Geier-Greguska, Fett/Lipid, 1996b, 98(7-8), 253-256.

22 M. E. Miquel, S. Carli, P. J. Couzens, H. J. Wille and L. D. Hall, Food Res. Int., 2001, 34(9), 773-781.

23 R. S. Khan and D. Rousseau, Eur. J. Lipid Sci. Technol., 2006, 108, 434-443.

24 N. L. Green and D. Rousseau, Soft Matter, 2015, 11, 5523-5530. 25 F. Maleky, A. Smith and A. G. Marangoni, Cryst. Growth Des., 2011, 11, 2335-2345.

26 J. Garside and R. J. Davey, Chem. Eng. Commun., 1980, 4, 393424. 
27 S. Sonwai and M. R. Mackley, J. Am. Oil Chem. Soc., 2006, 83, 583-596.

28 P. Walstra, W. Kloek and T. van Vliet, Crystallization Processes in Fats and Lipid Systems, Marcel Dekker Inc., New York, USA, 2006.
29 T. S. Omonov, L. Bouzidi and S. S. Narine, Chem. Phys. Lipids, 2010, 163, 728-740.

30 A. Bot, Y. S. J. Veldhuizen, R. den Adel and E. C. Roijers, Food Hydrocolloids, 2009, 23, 1184-1189. 